\title{
Effectiveness and Safety of Tranexamic Acid in Spinal Deformity Surgery
}

Ho Yong Choi, M.D., Seung-Jae Hyun, M.D., Ph.D., Ki-Jeong Kim, M.D., Ph.D., Tae-Ahn Jahng, M.D., Ph.D., Hyun-Jib Kim, M.D., Ph.D.

Department of Neurosurgery, Spine Center, Seoul National University Bundang Hospital, Seoul National University College of Medicine, Seongnam, Korea

Objective : Spinal deformity surgery has the potential risk of massive blood loss. To reduce surgical bleeding, the use of tranexamic acid (TXA) became popular in spinal surgery, recently. The purpose of this study was to determine the effectiveness of intraoperative TXA use to reduce surgical bleeding and transfusion requirements in spinal deformity surgery.

Methods : A total of 132 consecutive patients undergoing multi-level posterior spinal segmental instrumented fusion ( $\geq 5$ levels) were analyzed retrospectively. Primary outcome measures included intraoperative estimated blood loss (EBL), transfusion amount and rate of transfusion. Secondary outcome measures included postoperative transfusion amount, rate of transfusion, and complications associated with TXA or allogeneic blood transfusions.

Results : The number of patients was 89 in TXA group and 43 in non-TXA group. There were no significant differences in demographic or surgical traits between the groups except hypertension. The EBL was significantly lower in TXA group than nonTXA group (841 vs. $1336 \mathrm{~mL}, p=0.002$ ). TXA group also showed less intra-operative and postoperative transfusion requirements (544 vs. $812 \mathrm{~mL}, p=0.012 ; 193 \mathrm{vs.} 359 \mathrm{~mL}, p=0.034$ ). Based on multiple regression analysis, TXA use could reduce surgical bleeding by 371 $\mathrm{mL}$ ( $37 \%$ of mean EBL). Complication rate was not different between the groups.

Conclusion : TXA use can effectively reduce the amount of intra-operative bleeding and transfusion requirements in spinal deformity surgery. Future randomized controlled study could confirm the routine use of TXA in major spinal surgery.

Key Words : Tranexamic acid · Antifibrinolytics · Spinal surgery · Surgical blood loss.

\section{INTRODUCTION}

Spinal deformity surgery has the potential for massive blood loss ${ }^{15,19)}$. Especially during long level lumbar or thoracic instrumented fusion with or without osteotomy, blood loss may be substantial and transfusion is required in most cases ${ }^{11,12}$. Extensive blood loss can lead to massive blood transfusions, pulmonary or cerebral edema, and shock. Allogeneic blood transfusions are associated with blood-borne disease transmission, hemolytic and non-hemolytic transfusion reactions, and

- Received: May 16, 2016 • Revised: September 7, 2016 •Accepted: October 3, 2016

- Address for reprints : Seung-Jae Hyun, M.D., Ph.D.

Department of Neurosurgery, Spine Center, Seoul National University Bundang Hospital, Seoul National University College of Medicine, 82 Gumi-ro 173beon-gil, Bundang-gu, Seongnam 13620, Korea

Tel : +82-31-787-7164, Fax : +82-31-787-4059, E-mail : hyunsj@snu.ac.kr

This is an Open Access article distributed under the terms of the Creative Commons Attribution Non-Commercial License (http://creativecommons.org/licenses/by-nc/4.0) which permits unrestricted non-commercial use, distribution, and reproduction in any medium, provided the original work is properly cited. 
transfusion-related $\operatorname{cost}^{6,21)}$. Moreover, there are not a few literatures reporting allogeneic blood transfusions were related to not only wound infection but also overall postoperative infections $^{9,13)}$.

Therefore, there have been many options in blood conservation strategies to reduce surgical bleeding and intraoperative allogeneic blood transfusions. Such options include patients positioning to avoid abdominal compression, hypotensive anesthesia, application of topical hemostatic agents to the decorticated bone, intra-operative cell salvage (ICS) system, acute normovolemic hemodilution, and administration of medications ${ }^{2,3,10)}$.

Recently, the use of antifibrinolytics has become popular in major spinal surgeries. Tranexamic acid (TXA) is a synthetic antifibrinolytic amino acid derivative that forms a reversible complex with both plasminogen and plasmin by binding at lysine binding sites. This binding completely blocks the interaction of plasminogen and plasmin with lysine residues on the surface of fibrin, thereby preventing proteolytic action of plasmin on fibrin and inhibiting fibrinolysis at the surgical wound ${ }^{7)}$. Although several literatures about TXA use demonstrated favorable results in spinal surgery, there still exists inconsistency.

The purpose of this study was to evaluate the effectiveness and safety of using TXA for spinal deformity surgery. This study also explored factors related to intra-operative surgical bleeding.

\section{MATERIALS AND METHODS}

This study was approved by our hospital institutional review board before its commencement. A total number of 132 patients undergoing multi-level posterior spinal fusion $(\geq 5$ levels) with segmental instrumentation for spinal deformity between June 2010 and December 2015 at a single academic institution were enrolled and analyzed retrospectively. The patients were not randomized, however, consecutively enrolled.

Patients who underwent spinal fusion surgery including five segments or more, regardless of osteotomy, were included. Both revision and primary cases were included. Exclusion criteria were patients with infectious disease, spinal tumor including metastasis. Among 132 patients enrolled, the most common diagnosis was degenerative flatback (35 patients), followed by adolescent idiopathic scoliosis (23 patients), posttraumatic kyphosis (23 patients), iatrogenic flatback (14 patients), and degenerative lumbar scoliosis (10 patients). Less common etiology included junctional kyphosis (six patients), tuberculosis kyphosis (six patients), syndromic kyphosis (four patients), Scheuerman kyphosis (three patients), scoliosis associated with Chiari malformation (two patients), congenital scoliosis (two patients), post-laminectomy kyphosis (two patients), ankylosing spondylitis (one patient), and adult cervico-thoracic scoliosis (one patient).

Basically, decision to use TXA was determined by the extent of surgery. TXA was administered in most cases of spinal deformity surgery unless the patients had a history of thromboembolic events. Therefore, we did not use TXA in order to avoid thromboembolic complications if the patients had suffered from stroke, angina, myocardial infarction, pulmonary embolism, or deep vein thrombosis.

Demographic information collected included age, sex, body mass index, comorbidities, smoking status, primary or revision surgery status, and anti-platelet medication. Patients taking anti-platelet medication were instructed to stop taking medication for five days before surgery. Operative details assessed included conduction of osteotomy, the number of fused segments, the number of interbody fusion, estimated blood loss (EBL), postoperative drain amount, intra-/postoperative transfusion amount and rate, and surgical time. Intraoperative transfusion amount included both transfused volume by ICS system and allogeneic blood. Rate of intraoperative transfusion was also determined by any of autologous or allogeneic blood transfusion. However, postoperative blood transfusion was performed using allogeneic blood only. We also assessed perioperative hemoglobin levels and possible complications associated with TXA usage.

All patients underwent a similar operative technique by a single surgeon: Standard posterior subperiosteal exposure, 
facet release, screw insertion by free-hand technique, neural decompression, and transforaminal interbody fusion, pedicle subtraction osteotomy or posterior vertebral column resection, if needed. After rod assembly, posterior elements or transverse processes were decorticated, followed by posterior onlay fusion or posterolateral fusion in all patients. All wounds were closed with negative suction drain.

The treatment regimen of TXA was a loading dose more than 15 minutes before skin incision, followed by a maintenance dose for the duration of the surgery. The dose of TXA was dichotomized by patient's age. In adult patients $(\geq 18$ years old), a regimen of a loading dose of $10 \mathrm{mg} / \mathrm{kg}$, followed by a maintenance dose of $1 \mathrm{mg} / \mathrm{kg} / \mathrm{h}$ was used. In pediatric population ( $<18$ years old), a regimen of a loading dose of 50 $\mathrm{mg} / \mathrm{kg}$ and maintenance dose of $5 \mathrm{mg} / \mathrm{kg} / \mathrm{h}$ was utilized.

The same blood transfusion guidelines were used for all patients. Allogeneic blood transfusion was performed if hemoglobin decreased to $<7.0 \mathrm{mg} / \mathrm{dL}$ or if anemic symptoms developed, such as a decrease in blood pressure to $<100$ $\mathrm{mmHg}$ systolic, tachycardia greater than 100 beats/min, or a low urine output of $<30 \mathrm{~mL} / \mathrm{h}$, even after initial fluid challenge with $500 \mathrm{~mL}$ normal saline in patients with a hemoglobin level between 7.0 and $8.0 \mathrm{mg} / \mathrm{dL}$.

\section{Statistical analysis}

Using IBM SPSS 22.0 software for Windows (IBM Corp., Armonk, NY, USA), dichotomous data was compared using chi-square tests, whereas Student's $t$ test were used for comparisons of parametric and nonparametric data, respectively.
A stepwise multiple regression analysis was performed between selected covariates to investigate the predictive factors of EBL. Statistical significance was set at $p<0.05$.

\section{RESULTS}

Descriptive analysis was performed to summarize patient and surgical characteristics. Eighty-nine (67.4\%) patents were operated with administration of TXA, whereas forty-three (32.6\%) patients without TXA (Table 1). There were no significant differences in terms of age, sex, BMI, diabetes, smoking status, preoperative hemoglobin, and previous surgery status between two groups. The proportion of patients with underlying hypertension was higher in TXA group than in non-TXA group (36.0 vs. 58.1\%), and the difference was significant statistically ( $p=0.016$ ). The proportion of patients taking anti-platelet medication was higher in non-TXA group (19.1 vs. 30.2\%), however, the difference did not reach statistical significance $(p=0.185)$.

Operative characteristics were summarized in Table 2. The proportion of osteotomy was similar between TXA and nonTXA groups (39.3 vs. $37.2 \%$ ). The differences were not significant either, regarding the number of fused segments and the number of interbody fusion in both groups. Surgical time had a trend toward shorter in TXA group than in non-TXA group (370.28 vs. 397.86 minutes), however the difference did not reach statistical significance $(p=0.136)$.

Regarding surgical bleeding, TXA group was significantly

Table 1. Summary of demographic data

\begin{tabular}{|c|c|c|c|c|}
\hline & All patients $(n=132)$ & TXA group $(n=89)$ & Non-TXA group $(n=43)$ & $p$-value \\
\hline Age (yr) & $55.18 \pm 23.96$ & $53.15 \pm 24.75$ & $59.40 \pm 21.91$ & 0.145 \\
\hline Sex, male (\%) & 20.5 & 18.0 & 25.6 & 0.314 \\
\hline Body mass index $\left(\mathrm{kg} / \mathrm{m}^{2}\right)$ & $23.85 \pm 4.39$ & $24.30 \pm 4.74$ & $22.92 \pm 3.41$ & 0.091 \\
\hline Hypertension (\%) & 43.2 & 36.0 & 58.1 & 0.016 \\
\hline Diabetes (\%) & 14.4 & 15.7 & 11.6 & 0.533 \\
\hline Smoker (\%) & 3.8 & 4.5 & 2.3 & 0.544 \\
\hline Previous spine surgery (\%) & 32.6 & 34.8 & 27.9 & 0.430 \\
\hline Anti-platelet medication (\%) & 22.7 & 30.2 & 19.1 & 0.185 \\
\hline
\end{tabular}

Values are presented as \% or mean \pm standard deviation. TXA : tranexamic acid 
Table 2. Operative characteristics

\begin{tabular}{|c|c|c|c|}
\hline & TXA group $(n=89)$ & Non-TXA group $(n=43)$ & $p$-value \\
\hline Osteotomy (\%) & 39.3 & 37.2 & 0.817 \\
\hline Number of fused segments & $9.38 \pm 2.91$ & $9.81 \pm 3.54$ & 0.459 \\
\hline Number of interbody fusion & $0.63 \pm 0.83$ & $0.88 \pm 1.22$ & 0.221 \\
\hline Estimated blood loss (mL) & $841.01 \pm 559.55$ & $1336.05 \pm 923.36$ & 0.002 \\
\hline Postoperative drain amount & $1041.27 \pm 41.84$ & $1142.28 \pm 75.46$ & 0.208 \\
\hline \multicolumn{4}{|l|}{ Intraoperative transfusion } \\
\hline Transfusion amount (mL) & $544.21 \pm 532.55$ & $812.30 \pm 625.20$ & 0.012 \\
\hline Rate of transfusion (\%) & 74.2 & 88.4 & 0.039 \\
\hline \multicolumn{4}{|l|}{ Postoperative transfusion } \\
\hline Transfusion amount (mL) & $192.65 \pm 263.38$ & $359.33 \pm 468.29$ & 0.034 \\
\hline Rate of transfusion (\%) & 41.6 & 51.2 & 0.303 \\
\hline Surgical time (min) & $370.28 \pm 114.58$ & $397.86 \pm 136.46$ & 0.136 \\
\hline
\end{tabular}

Values are presented as \% or mean \pm standard deviation. TXA : tranexamic acid

Table 3. Changes in hemoglobin level

\begin{tabular}{|c|c|c|c|}
\hline & TXA group $(n=89)$ & Non-TXA group $(n=43)$ & $p$-value \\
\hline Hemoglobin level (g/dL) & & & 0.762 \\
\hline Preoperative & $12.97 \pm 1.73$ & $13.03 \pm 1.73$ & \\
\hline Postoperative day 1 & $10.70 \pm 1.55$ & $10.53 \pm 1.58$ & \\
\hline Postoperative day 2 & $10.36 \pm 1.44$ & $10.18 \pm 1.07$ & \\
\hline
\end{tabular}

Values are presented as mean \pm standard deviation. TXA : tranexamic acid

associated with less amount of EBL than control group (841.01 vs. $1336.05 \mathrm{~mL}, p=0.002$ ). Intra-operative transfusion amount and the rate were also significantly lower in TXA group than non-TXA group (544.21 vs. $812.30 \mathrm{~mL}, p=0.012$; 74.2 vs. $88.4 \%, p=0.039$ ).

Upon variables associated with postoperative bleeding, postoperative drain amount showed a tendency toward lower in TXA group $(1041.27 \mathrm{~mL})$ than that in non-TXA group $(1142.28 \mathrm{~mL})$, but the difference did not reach statistical significance $(p=0.208)$. Postoperative transfusion amount was $192.65 \mathrm{~mL}$ in TXA group, whereas $359.33 \mathrm{~mL}$ in non-TXA group, and the difference was significant $(p=0.034)$. The rate of postoperative transfusion was also lower in TXA group than control, however, the difference was not significant statistically (41.6 vs. $51.2 \%, p=0.303$ ).

\section{Changes in hemoglobin level}

In order to assess perioperative changes and differences in hemoglobin levels between the groups, analysis of variance was conducted. Routine hemoglobin levels were checked three times; preoperatively, postoperative day 1 , and postoperative day 2 (Table 3). In TXA group, preoperative hemoglobin level was slightly lower (12.97 vs. $13.03 \mathrm{~g} / \mathrm{dL}$ ), whereas slightly higher at postoperative day 1 (10.70 vs. $10.53 \mathrm{~g} / \mathrm{dL}$ ), and day 2 (10.36 vs. $10.18 \mathrm{~g} / \mathrm{dL}$ ). The differences were not significant between groups ( $p=0.762$ ).

\section{Predicting amount of surgical bleeding}

To find out independent factors associated with surgical bleeding, multiple stepwise regression analysis was conducted upon variables, which showed statistically significance on correlation analysis of Pearson, including age, height, BMI, conduction of osteotomy, number of fused segments, number of interbody fusion, surgical time, and preoperative hemoglobin level. Use of TXA was negatively correlated with EBL $(p=0.000)$, whereas surgical time was positively corre- 
Table 4. Results of stepwise multiple regression analysis with estimated blood loss as a dependent variable

\begin{tabular}{lccccc}
\hline & Regression coefficient & Standard error & T stat & $\boldsymbol{p}$-value & $\mathbf{9 5} \% \mathbf{C l}$ \\
\hline Surgical time (hours) & 3.66 & 0.39 & 9.45 & 0.000 & $2.89-4.42$ \\
TXA use & -370.62 & 100.93 & -3.67 & 0.000 & $-570.31--170.92$ \\
\hline
\end{tabular}

$\mathrm{R}^{2}=0.47 . \mathrm{Cl}$ : confidence interval, TXA : tranexamic acid

lated with EBL ( $p=0.000$ ) (Table 4). According to the regression analysis, use of TXA decreased the amount of surgical bleeding by $371 \mathrm{~mL}$, which was about 37\% of mean EBL (1002.27 mL).

\section{Complications associated with TXA or allogeneic blood transfusions}

Potential complications related to TXA or allogeneic blood transfusions were summarized in Table 5. Each angina and myocardial infarction happened in postoperative period, only in non-TXA group. There were cases of one deep vein thrombosis and one pulmonary thromboembolism in TXA group, but no statistical significance was noted. There was no case of acute kidney injury postoperatively. Regarding allergic reaction, electrolyte changes, elevated liver enzymes, new onset arrhythmia, pneumonia, wound problem, and urinary tract infection, there were no significant differences between two groups.

\section{DISCUSSION}

In the present study, we found the use of TXA was effective in reducing surgical bleeding, intra-operative transfusion requirements, and postoperative transfusion volume for patients undergoing spinal deformity surgeries. Also, there was a tendency toward lower postoperative transfusion rate and less postoperative drain amount in TXA group, although the differences did not reach statistical significance. These results were concordant with the majority of previous studies and recently published meta-analysis ${ }^{5,22,23,27)}$.

Plenty of studies demonstrated the efficacy of TXA use in reducing intra-operative blood loss in spinal surgeries. Previous researcher stated in their randomized prospective study that TXA use decreased surgical bleeding by $25 \%$ in adult pa-
Table 5. Summary of possible complications associated with TXA or transfusions

\begin{tabular}{lccc}
\hline & $\begin{array}{c}\text { TXA group } \\
(\mathbf{n = 8 9 )}\end{array}$ & $\begin{array}{c}\text { Non-TXA group } \\
(\mathbf{n = 4 3 )}\end{array}$ & $\boldsymbol{p}$-value \\
\hline Allergic reaction & $2.2(2)$ & $0(0)$ & 1.000 \\
Electrolyte changes & $16.9(15)$ & $20.9(9)$ & 0.569 \\
Elevated liver enzymes & $32.6(29)$ & $23.3(10)$ & 0.271 \\
\hline Angina & $0(0)$ & $2.3(1)$ & 0.326 \\
Myocardial infarction & $0(0)$ & $2.3(1)$ & 0.326 \\
New onset arrhythmia & $2.2(2)$ & $2.3(1)$ & 1.000 \\
Pneumonia & $1.1(1)$ & $2.3(1)$ & 0.547 \\
DVT/PTE & $2.2(2)$ & $0(0)$ & 1.000 \\
Wound problem & $4.5(4)$ & $2.3(1)$ & 1.000 \\
Urinary tract infection & $1.1(1)$ & $2.3(1)$ & 0.547 \\
Acute kidney injury & $0(0)$ & $0(0)$ & - \\
\hline
\end{tabular}

Values are presented as \% (number). TXA : tranexamic acid, DVT : deep vein thrombosis, PTE : pulmonary thromboembolism

tients having spinal fusion surgery ${ }^{25)}$. Xie and colleagues also reported less surgical bleeding and transfusion amount in TXA group without increased rate of complications in spine correction surgery $^{26)}$. Moreover, another investigator notified less surgical time in TXA group in addition to the benefit of reduced surgical bleeding and transfusion amount ${ }^{18)}$.

On the other hand, literatures reporting lack of efficacy of TXA use also exist. Previous investigator did not find any significant differences in intraoperative blood losses or transfusion requirements between TXA and control groups in patients with spinal deformity ${ }^{17,20)}$. Baldus and colleagues concluded in their study in patients undergoing lumbar pedicle subtraction osteotomy that TXA use did not decrease surgical bleeding or intraoperative transfusion amount whereas using aprotinin $\mathrm{did}^{1)}$. These inconsistent results may warrant more number of future refined studies, despite most high level of evidence studies favor the use of TXA in spinal surgery $^{5,24,25)}$. Most studies showed tendency toward less postoperative bleeding in TXA group with or without statistical significance ${ }^{1,20,24,25)}$. Present study also demonstrated less postoperative drain amount in TXA group without reaching 
statistical significance. The effect estimate of TXA was-605 $\mathrm{mL}$ for intraoperative bleeding, whereas $-361 \mathrm{~mL}$ for postoperative blood loss in previous meta-analysis ${ }^{23)}$. This less degree of impact on reducing postoperative bleeding of TXA compared to intraoperative bleeding could result in inconsistent statistical significances among studies.

There were no differences in hemoglobin levels between two groups. Previous studies reported inconsistent results regarding hematologic profiles. Some researchers found that postoperative hematocrit was significant higher in TXA group than control group ${ }^{8,25)}$. On the other hand, we found only statistically insignificant trend toward higher postoperative hemoglobin levels in TXA group, which was in accordance with the results described by Peters and colleagues ${ }^{20)}$. In this study, postoperative hemoglobin level was almost similar between groups in spite of larger blood loss in nonTXA group. This result may be attributed to larger transfused volume intraoperatively, by application of same blood transfusion guideline (described above) to maintain adequate intravascular volume.

Another issue with the use of TXA is about safety concerns. The primary concern with the use of TXA is the potential for an increased risk of thromboembolic complications such as peri-operative myocardial infarction, stroke, deep vein thrombosis, and pulmonary embolism ${ }^{4,25)}$. Also, there have been reports that use of high-dose TXA resulted in seizure in patients undergoing cardiac surgery ${ }^{14,16)}$. However, there has been no case reporting seizure associated with TXA use in patients having spine surgery, and recent literatures including meta-analysis demonstrated that TXA was not associated with increased incidence of such thromboembolic complications ${ }^{5,23)}$. The present study also showed that complication rate was not different between TXA group and control group.

The potential weakness of this study is that it was retrospective and non-randomized. When large amount of surgical bleeding was expected, however, it was reasonable to use TXA to avoid substantial blood loss and consequential serious complications. In fact, there were no significant differences in demographic data and surgical traits between two groups. The only difference was higher proportion of hypertension in non-TXA group, which might reflect higher incidence of past medical history of thromboembolic events that precluded the use of TXA. Furthermore, after adjusting potential bias and confounding variables, using TXA was still efficacious in decreasing intra-operative blood loss.

Another weakness was the heterogeneity of dose of TXA between adult and pediatric patients. TXA was administered to adult patients with lower dose, whereas pediatric patients with higher dose. It was because of the concerns about potential complications of thromboembolic events associated with TXA in older populations with higher comorbidities. Despite studies reporting similar efficacy in reducing surgical bleeding and transfusion requirements in both low and high dose regimen ${ }^{5}$, unification of dosage would have drawn more refined results.

\section{CONCLUSION}

For spinal deformity surgery, use of TXA can provide beneficial effects not only on reducing surgical bleeding, but also on decreasing transfusion requirements both intra- and postoperatively. Incidence of thromboembolic event and other complications did not show any differences between TXA and non-TXA groups. Future prospective randomized study will be needed to ascertain the efficacy of routine use of TXA in major spinal surgery.

\section{References}

1. Baldus CR, Bridwell KH, Lenke LG, Okubadejo GO : Can we safely reduce blood loss during lumbar pedicle subtraction osteotomy procedures using tranexamic acid or aprotinin? A comparative study with controls. Spine (Phila Pa 1976) 35 : 235-239, 2010

2. Bess RS, Lenke LG, Bridwell KH, Steger-May K, Hensley M : Wasting of preoperatively donated autologous blood in the surgical treatment of adolescent idiopathic scoliosis. Spine (Phila Pa 1976) 31 : 23752380, 2006

3. Cardone D, Klein AA : Perioperative blood conservation. Eur J Anaesthesiol $26: 722-729,2009$ 
4. Casati V, Della Valle P, Benussi S, Franco A, Gerli C, Baili P, et al. : Effects of tranexamic acid on postoperative bleeding and related hematochemical variables in coronary surgery: Comparison between on-pump and off-pump techniques. J Thorac Cardiovasc Surg 128: 83-91, 2004

5. Cheriyan T, Maier SP 2nd, Bianco K, Slobodyanyuk K, Rattenni RN, Lafage $V$, et al. : Efficacy of tranexamic acid on surgical bleeding in spine surgery: a meta-analysis. Spine J 15 : 752-761, 2015

6. Despotis GJ, Avidan MS, Hogue CW Jr : Mechanisms and attenuation of hemostatic activation during extracorporeal circulation. Ann Thorac Surg 72 : S1821-S1831, 2001

7. Dunn CJ, Goa KL: Tranexamic acid: a review of its use in surgery and other indications. Drugs 57 : 1005-1032, 1999

8. Elwatidy S, Jamjoom Z, Elgamal E, Zakaria A, Turkistani A, El-Dawlatly A : Efficacy and safety of prophylactic large dose of tranexamic acid in spine surgery: a prospective, randomized, double-blind, placebocontrolled study. Spine (Phila Pa 1976) 33 : 2577-2580, 2008

9. Friedman $R$, Homering M, Holberg G, Berkowitz SD : Allogeneic blood transfusions and postoperative infections after total hip or knee arthroplasty. J Bone Joint Surg Am 96 : 272-278, 2014

10. Gill JB, Chin $Y$, Levin $A$, Feng $D$ : The use of antifibrinolytic agents in spine surgery. A meta-analysis. J Bone Joint Surg Am 90 : 23992407, 2008

11. Hyun SJ, Kim YJ, Rhim SC : Spinal pedicle subtraction osteotomy for fixed sagittal imbalance patients. World J Clin Cases 1 : 242-248, 2013

12. Hyun SJ, Rhim SC : Clinical outcomes and complications after pedicle subtraction osteotomy for fixed sagittal imbalance patients : a long-term follow-up data. J Korean Neurosurg Soc 47 : 95-101, 2010

13. Janssen SJ, Braun Y, Wood KB, Cha TD, Schwab JH : Allogeneic blood transfusions and postoperative infections after lumbar spine surgery. Spine J 15 : 901-909, 2015

14. Keyl C, Uhl R, Beyersdorf F, Stampf S, Lehane C, Wiesenack C, et al. : High-dose tranexamic acid is related to increased risk of generalized seizures after aortic valve replacement. Eur J Cardiothorac Surg 39 : e114-e121, 2011

15. Lentschener C, Cottin P, Bouaziz H, Mercier FJ, Wolf M, Aljabi Y, et al. : Reduction of blood loss and transfusion requirement by aprotinin in posterior lumbar spine fusion. Anesth Analg 89 : 590-597, 1999

16. Martin K, Knorr J, Breuer T, Gertler R, Macguill M, Lange R, et al. : Seizures after open heart surgery: comparison of epsilon-aminocaproic acid and tranexamic acid. J Cardiothorac Vasc Anesth 25 : 20-25, 2011

17. Neilipovitz DT, Murto K, Hall L, Barrowman NJ, Splinter WM : A randomized trial of tranexamic acid to reduce blood transfusion for scoliosis surgery. Anesth Analg 93 : 82-87, 2001

18. Ng BK, Chau WW, Hung AL, Hui AC, Lam TP, Cheng JC: Use of Tranexamic Acid (TXA) on reducing blood loss during scoliosis surgery in Chinese adolescents. Scoliosis $10: 28,2015$

19. Nuttall GA, Horlocker TT, Santrach PJ, Oliver WC Jr, Dekutoski MB, Bryant $S$ : Predictors of blood transfusions in spinal instrumentation and fusion surgery. Spine (Phila Pa 1976) 25 : 596-601, 2000

20. Peters A, Verma K, Slobodyanyuk K, Cheriyan T, Hoelscher C, Schwab $F$, et al. : Antifibrinolytics reduce blood loss in adult spinal deformity surgery: a prospective, randomized controlled trial. Spine (Phila Pa 1976) 40 : E443-E449, 2015

21. Urban MK, Beckman J, Gordon M, Urquhart B, Boachie-Adjei 0 : The efficacy of antifibrinolytics in the reduction of blood loss during complex adult reconstructive spine surgery. Spine (Phila Pa 1976) : 1152-1156, 2001

22. Verma K, Errico T, Diefenbach C, Hoelscher C, Peters A, Dryer J, et al. : The relative efficacy of antifibrinolytics in adolescent idiopathic scoliosis: a prospective randomized trial. J Bone Joint Surg Am 96 : e80, 2014

23. Wang $M$, Zheng $X F$, Jiang $L S$ : Efficacy and safety of antifibrinolytic agents in reducing perioperative blood loss and transfusion requirements in scoliosis surgery: a systematic review and meta-analysis. PLoS One 10 : e0137886, 2015

24. Wang Q, Liu J, Fan R, Chen Y, Yu H, Bi Y, et al. : Tranexamic acid reduces postoperative blood loss of degenerative lumbar instability with stenosis in posterior approach lumbar surgery: a randomized controlled trial. Eur Spine J 22 : 2035-2038, 2013

25. Wong J, El Beheiry H, Rampersaud YR, Lewis S, Ahn H, De Silva Y, et al. : Tranexamic Acid reduces perioperative blood loss in adult patients having spinal fusion surgery. Anesth Analg 107 : 1479-1486, 2008

26. Xie J, Lenke LG, Li T, Si Y, Zhao Z, Wang Y, et al. : Preliminary investigation of high-dose tranexamic acid for controlling intraoperative blood loss in patients undergoing spine correction surgery. Spine J 15 : 647654,2015

27. Yagi M, Hasegawa J, Nagoshi N, lizuka S, Kaneko S, Fukuda K, et al. : Does the intraoperative tranexamic acid decrease operative blood loss during posterior spinal fusion for treatment of adolescent idiopathic scoliosis? Spine (Phila Pa 1976) 37 : E1336-E1342, 2012 